\title{
Efficient Nonuniform Schemes for Paraxial and Wide-Angle Finite-Difference Beam Propagation Methods
}

\author{
Jun Shibayama, Member, OSA, Kenji Matsubara, Minoru Sekiguchi, Junji Yamauchi, Member, IEEE, Member, OSA, \\ and Hisamatsu Nakano, Fellow, IEEE
}

\begin{abstract}
Efficient nonuniform schemes, based on the generalized Douglas (GD) scheme, are developed for the finite-difference beam propagation method (FD-BPM). For a two-dimensional (2D) problem, two methods are presented: a computational space method and a physical space method. In the former, the GD scheme is employed, after replacing a nonuniform grid in the physical space with a uniform one in the computational space. In the latter, the GD scheme is directly extended to a nonuniform grid in the physical space. We apply these two methods to paraxial and wide-angle FD-BPM's. The fourth-order accuracy is achieved in the transverse direction, provided that the grid growth factor between two adjacent grids is $r=1+O(\Delta x)$. For the paraxial BPM, the reduction in the truncation error is demonstrated through modal calculations of a graded-index waveguide using an imaginary distance procedure. For the wide-angle BPM, the propagating field in a tilted waveguide is analyzed to show the effectiveness of the present scheme. As an application of the physical space method, an adaptive grid is introduced into the multistep method.
\end{abstract}

Index Terms - Finite-difference methods, optical beam propagation, optical waveguides.

\section{INTRODUCTION}

W AVE propagation in an optical waveguide can be analyzed by the beam propagation method (BPM) [1], [2]. The BPM's developed during the last two decades can be roughly categorized in terms of the discretization in the transverse direction, i.e., fast Fourier transform (FFT-BPM) [3], finite-difference (FD-BPM) [4]-[10], finite-element (FEBPM) [11]-[14], and method of lines (Mol-BPM) [15]. In the FFT-BPM only a uniform grid is valid, while in the other BPM's a nonuniform grid [16]-[25] is allowed to improve computational efficiency or describe a waveguide configuration accurately. For a longitudinally invariant waveguide, a nonuniform grid has been applied to the FD-BPM [16], [17], [20]. In the case of a longitudinally variant waveguide, an adaptive grid has been introduced into the FD-BPM [18], [19], [21] and the FE-BPM [22], [24], [25].

For the FD-BPM, most of the previous works related to a nonuniform scheme have been based on the conventional nonuniform Crank-Nicholson (CN) scheme [17], [19], [20]. Accordingly, the truncation error is $O(\Delta x)^{2}$, provided that the grid growth factor between two adjacent grids is $r=1+O(\Delta x)$. Yevick et al. [16] refined the conventional

Manuscript received January 22, 1998; revised December 3, 1998.

The authors are with the College of Engineering, Hosei University, Tokyo 184-8584 Japan.

Publisher Item Identifier S 0733-8724(99)02697-3. nonuniform operator of the second derivative and improved its accuracy. Unfortunately, this results in a pentadiagonal matrix with time-consuming procedures. On the other hand, by eliminating the low-order error terms in a Taylor-series expansion, we have demonstrated that the generalized Douglas (GD) scheme [26]-[28] can be extended to a nonuniform grid [29], maintaining a tridiagonal matrix. It is worth mentioning that the truncation error, even in a nonuniform grid, can be reduced to $O(\Delta x)^{4}$ under the condition of $r=1+O(\Delta x)$. More recently, Vassallo and Van der Keur derived another nonuniform scheme whose truncation error is $O(\Delta x)^{3}$ [30].

As an alternative to treat a nonuniform grid, a mapping technique can be utilized to transform a nonuniform grid in the physical space into a uniform one in the computational space. Ladouceur [31] first applied this technique to the BPM analysis with the intention of avoiding a spurious reflection from the computational window edge. However, this technique reported so far has been based on the conventional $\mathrm{CN}$ scheme.

In this paper, we study the application of the GD scheme to an FD-BPM with a nonuniform grid for the purpose of developing efficient schemes for the modal and propagating-beam analyses. Two different methods are derived and discussed in a two-dimensional (2-D) problem. One is the computational space method (CSM) using a mapping technique [31]-[33], in which the GD scheme is employed after replacing a nonuniform grid in the physical space with a uniform one in the computational space. The other is the physical space method (PSM), in which the GD scheme is directly extended to a nonuniform grid in the physical space [29]. These two methods have the following feature: The CSM is easy to implement the algorithm since the transverse coordinate in the computational space is discretized by a uniform grid, while the grid growth factor is limited due to the mapping function to be used. The PSM is more flexible in the choice of the grid growth factor: An arbitrary region in the transverse coordinate can be densely or coarsely discretized.

The CSM and PSM are applied to paraxial and wideangle multistep [10], [34], [35] FD-BPM's (note that the multistep method based on the FE-BPM has been reported, but the use of a nonuniform grid has not been discussed [36]). To demonstrate the reduction in the truncation error in the paraxial FD-BPM, the modal analysis of a graded-index slab waveguide is carried out using the imaginary distance procedure [37]-[39]. For the assessment of the wide-angle 
BPM, the propagation of the fundamental mode is analyzed in a tilted slab waveguide with a graded-index profile.

As an application, taking advantage of the PSM, we introduce an adaptive grid [18], [19], [21], [22], [24], [25] into the multistep method for wide-angle beam propagation. A Lagrange interpolation technique is used to determine missing values between sampling points. The use of the multistep method combined with the adaptive grid can substantially reduce the total number of the transverse sampling points, without losing inherent accuracy.

\section{FORMULATION}

Throughout this paper, we consider a 2-D problem. We express the scalar field $e(x, z)$ as $e(x, z)=$ $E(x, z) \exp \left(-j k n_{0} z\right)$, so that the wave equation to be solved is

$$
\sigma \frac{\partial E(x, z)}{\partial z}=\frac{\partial^{2} E(x, z)}{\partial z^{2}}+\frac{\partial^{2} E(x, z)}{\partial x^{2}}+\nu E(x, z)
$$

where $\sigma=2 j k n_{0}$ and $\nu=k^{2}\left[n^{2}(x, z)-n_{0}^{2}\right]$, in which $k$ is the free space wavenumber, $n(x, z)$ is the index profile of the waveguide, and $n_{0}$ is the reference index to be appropriately chosen. For the paraxial formulation, the second derivative with respect to $z$ is omitted. This leads to the Fresnel equation. For the wide-angle formulation, the second derivative is partially taken into account using a Padé approximation [8], [9]. With the use of the multistep method [34], the higher Padé approximant operator is factored into a series of simpler Padé $(1,1)$ operators.

\section{A. Computational Space Method (CSM)}

We begin with the formulation of the CSM. A nonuniform grid in the physical space $(x, z)$ is transformed into a uniform grid in the computational space $(u, z)$ using a mapping function. In this paper, we choose the mapping function defined as [31]

$$
x=\alpha \tan (u)
$$

where $\alpha$ is a scaling parameter. Although alternative mapping functions can be chosen, (2) leads to a simplified expression of the wave equation, as will be seen in (4). For (2), the sampling width in the physical space increases gradually from the center of the computational region to the edge. This type of grid growth is particularly suitable for a modal analysis of an optical waveguide, because the region with a large field amplitude, which predominates the accuracy, is densely discretized, while the region with a small field amplitude is coarsely discretized. Equation (2) has been successfully used for the eigenmode analysis of optical waveguides [32], [33].

To facilitate derivation of the finite-difference equation, the transformation of the field

$$
E(x, z)=\mathcal{E}(u, z) / \cos (u)
$$

is imposed. Using (2) and (3), (1) can be rewritten as follows:

$$
\sigma \frac{\partial \mathcal{E}(u, z)}{\partial z}=\frac{\partial^{2} \mathcal{E}(u, z)}{\partial z^{2}}+\frac{\cos ^{4}(u)}{\alpha^{2}}\left[\frac{\partial^{2} \mathcal{E}(u, z)}{\partial u^{2}}+\tau \mathcal{E}(u, z)\right]
$$

with

$$
\tau=1+\frac{\alpha^{2}}{\cos ^{4}(u)} \nu .
$$

For the paraxial formulation, we drop the second derivative with respect to $z$ in (4). Here we employ the GD scheme [26], [27] and evaluate the second derivative with respect to $u$ more precisely than the conventional three-point approximation. Since the transverse coordinate is uniformly discretized in the computational space, the derivation of the improved formula is straightforward. Finally, the following paraxial equation is obtained:

$$
\begin{aligned}
& f_{i+1}^{-} \mathcal{E}_{i+1}^{m+1}+g_{i}^{-} \mathcal{E}_{i}^{m+1}+f_{i-1}^{-} \mathcal{E}_{i-1}^{m+1} \\
& \quad=f_{i+1}^{+} \mathcal{E}_{i+1}^{m}+g_{i}^{+} \mathcal{E}_{i}^{m}+f_{i-1}^{+} \mathcal{E}_{i-1}^{m}
\end{aligned}
$$

where

$$
\begin{aligned}
f_{i \pm 1}^{ \pm} & =\frac{1}{12} \sigma \frac{\alpha^{2}}{\cos ^{4}\left(u_{i \pm 1}\right)} \pm \frac{\Delta z}{2 \Delta u^{2}} \pm \frac{\Delta z}{24} \tau_{i \pm 1} \\
g_{i}^{ \pm} & =\frac{5}{6} \sigma \frac{\alpha^{2}}{\cos ^{4}\left(u_{i}\right)} \mp \frac{\Delta z}{\Delta u^{2}} \pm \frac{5 \Delta z}{12} \tau_{i}
\end{aligned}
$$

and the superscript $m$ indicates position along the $z$ axis.

Consideration is next given to a wide-angle formulation. The second derivative with respect to $z$ in (4) is partially taken into account using a Padé recurrence relation. An $N$ th-order Padé operator may be decomposed into an $N$-step algorithm for which the $K$ th partial step takes the form [34]

$$
\mathcal{E}^{m+K / N}=\frac{1+a_{K} \mathcal{P}}{1+a_{K}^{*} \mathcal{P}} \mathcal{E}^{m+(K-1) / N}
$$

in which $\mathcal{P}=\cos ^{4}(u) / \alpha^{2}\left[\partial^{2} / \partial u^{2}+\tau\right]$. The $a$ 's can be determined by the one-time solution of an $N$ th-order complex algebraic equation. Equation (6) can be restored to the following differential equation:

$$
\frac{\partial \mathcal{E}}{\partial z}=\frac{2 j \operatorname{Im}\left\{a_{K}\right\} \mathcal{P}}{\left(1+\operatorname{Re}\left\{a_{K}\right\} \mathcal{P}\right) \Delta z / N} \mathcal{E}
$$

We apply the GD scheme to (7). Following the procedure described in [28] and [35], we finally obtain

$$
\begin{aligned}
& F_{i+1}^{m+K / N} \mathcal{E}_{i+1}^{m+K / N}+G_{i}^{m+K / N} \mathcal{E}_{i}^{m+K / N}+F_{i-1}^{m+K / N} \mathcal{E}_{i-1}^{m+K / N} \\
& \quad=F_{i+1}^{m+(K-1) / N} \mathcal{E}_{i+1}^{m+(K-1) / N}+G_{i}^{m+(K-1) / N} \mathcal{E}_{i}^{m+(K-1) / N} \\
& \quad+F_{i-1}^{m+(K-1) / N} \mathcal{E}_{i-1}^{m+(K-1) / N}
\end{aligned}
$$

where

$$
\begin{aligned}
F_{i \pm 1}^{m+(K-1) / N} & =\frac{1}{12} \frac{\alpha^{2}}{\cos ^{4}\left(u_{i \pm 1}\right)}+\Gamma_{K}\left(\frac{1}{\Delta u^{2}}+\frac{1}{12} \tau_{i \pm 1}^{m+1 / 2}\right) \\
G_{i}^{m+(K-1) / N} & =\frac{5}{6} \frac{\alpha^{2}}{\cos ^{4}\left(u_{i}\right)}+\Gamma_{K}\left(-\frac{2}{\Delta u^{2}}+\frac{5}{6} \tau_{i}^{m+1 / 2}\right)
\end{aligned}
$$

in which

$$
\begin{aligned}
& \Gamma_{K}=a_{K} \quad \text { for } \quad m+(K-1) / N \\
& =a_{K}^{*} \text { for } m+K / N \text {. }
\end{aligned}
$$

Evidently, (5) and (8) can be solved by the standard techniques, such as the Thomas algorithm. An advantage is found in the fact that the transverse coordinate is discretized by a 
uniform grid. This means that the solution of (5) and (8) can readily be achieved with only slight modification to the conventional algorithm. It should be noted, however, that the grid growth factor in the physical space is limited due to the mapping function to be used.

\section{B. Physical Space Method (PSM)}

Next we consider the direct extension of the GD scheme to a nonuniform grid. Since the formulation for the Fresnel equation has been briefly reported in [29], we here present more detailed derivation.

Consider the nonuniform grid shown in Fig. 1, where $r$ represents the grid growth factor. Note that $r$ can be larger or less than unity. By Taylor-series expansions, $E_{i+1}$ and $E_{i-1}$ are expressed as

$$
\begin{aligned}
E_{i+1}= & E_{i}+r \Delta x \frac{\partial E_{i}}{\partial x}+\frac{1}{2} r^{2} \Delta x^{2} \frac{\partial^{2} E_{i}}{\partial x^{2}}+\frac{1}{6} r^{3} \Delta x^{3} \frac{\partial^{3} E_{i}}{\partial x^{3}} \\
& +\frac{1}{24} r^{4} \Delta x^{4} \frac{\partial^{4} E_{i}}{\partial x^{4}}+\frac{1}{120} r^{5} \Delta x^{5} \frac{\partial^{5} E_{i}}{\partial x^{5}}+O(\Delta x)^{6} \\
E_{i-1}= & E_{i}-\Delta x \frac{\partial E_{i}}{\partial x}+\frac{1}{2} \Delta x^{2} \frac{\partial^{2} E_{i}}{\partial x^{2}}-\frac{1}{6} \Delta x^{3} \frac{\partial^{3} E_{i}}{\partial x^{3}} \\
& +\frac{1}{24} \Delta x^{4} \frac{\partial^{4} E_{i}}{\partial x^{4}}-\frac{1}{120} \Delta x^{5} \frac{\partial^{5} E_{i}}{\partial x^{5}}+O(\Delta x)^{6} .
\end{aligned}
$$

From (9) and (10), we have

$$
\begin{aligned}
\frac{\partial^{2} E_{i}}{\partial x^{2}}= & \frac{2}{\left(r^{2}+1\right) \Delta x^{2}} \\
& {\left[E_{i+1}-2 E_{i}+E_{i-1}-(r-1) \Delta x \frac{\partial E_{i}}{\partial x}\right.} \\
& -\frac{1}{6}\left(r^{3}-1\right) \Delta x^{3} \frac{\partial^{3} E_{i}}{\partial x^{3}}-\frac{1}{24}\left(r^{4}+1\right) \Delta x^{4} \frac{\partial^{4} E_{i}}{\partial x^{4}} \\
& \left.-\frac{1}{120}\left(r^{5}-1\right) \Delta x^{5} \frac{\partial^{5} E_{i}}{\partial x^{5}}\right] \\
& +O(\Delta x)^{4} \\
\frac{\partial E_{i}}{\partial x}= & \frac{\delta E_{i}}{\Delta x}-\frac{1}{2}(r-1) \Delta x \frac{\partial^{2} E_{i}}{\partial x^{2}}-\frac{1}{6}\left(r^{2}-r+1\right) \Delta x^{2} \frac{\partial^{3} E_{i}}{\partial x^{3}} \\
& -\frac{1}{24}\left(r^{2}+1\right)(r-1) \Delta x^{3} \frac{\partial^{4} E_{i}}{\partial x^{4}} \\
& -\frac{1}{120}\left(r^{4}-r^{3}+r^{2}-r+1\right) \Delta x^{4} \frac{\partial^{5} E_{i}}{\partial x^{5}}+O(\Delta x)^{5}
\end{aligned}
$$

where

$$
\delta E_{i}=\frac{E_{i+1}-E_{i-1}}{r+1} .
$$

By substituting (12) into (11), the second derivative can be written in the form

$$
\begin{aligned}
\frac{\partial^{2} E_{i}}{\partial x^{2}}= & \frac{\delta^{2} E_{i}}{\Delta x^{2}}-\frac{1}{3}(r-1) \Delta x \frac{\partial^{3} E_{i}}{\partial x^{3}} \\
& -\frac{1}{12}\left(r^{2}-r+1\right) \Delta x^{2} \frac{\partial^{4} E_{i}}{\partial x^{4}} \\
& -\frac{1}{60}(r-1)\left(r^{2}+r+1\right) \Delta x^{3} \frac{\partial^{5} E_{i}}{\partial x^{5}}+O(\Delta x)^{4}
\end{aligned}
$$

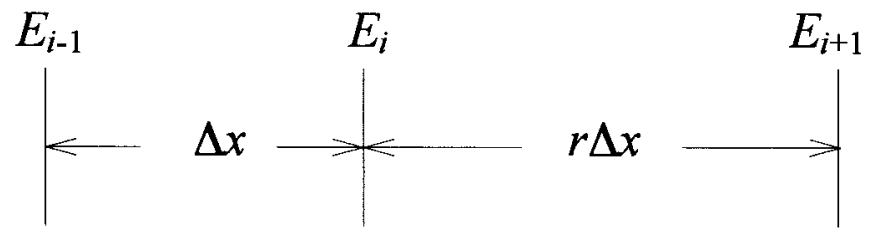

Fig. 1. Nonuniform sampling grid.

where

$$
\delta^{2} E_{i}=\frac{2}{r+1}\left[E_{i-1}-(1+1 / r) E_{i}+E_{i+1} / r\right] .
$$

It can be clearly seen that the first terms in the right-hand sides of (12) and (14) are the conventional nonuniform approximations to the first and second derivatives, respectively. Equation (15) has widely been used to analyze an optical waveguide [17], [19], [20]. Note that (13) and (15) have a truncation error of $O(\Delta x)^{2}$ under the condition of $r=1+O(\Delta x)$. We now evaluate the third and fourth derivatives in (14). Using the GD scheme, these derivatives are rewritten as follows:

$$
\begin{aligned}
& \frac{\partial^{3} E_{i}}{\partial x^{3}}=\frac{\delta}{\Delta x}\left(\sigma \frac{\partial E}{\partial z}-\nu E\right)_{i} \\
& \frac{\partial^{4} E_{i}}{\partial x^{4}}=\frac{\delta^{2}}{\Delta x^{2}}\left(\sigma \frac{\partial E}{\partial z}-\nu E\right)_{i} .
\end{aligned}
$$

Both (16) and (17) have a truncation error of $O(\Delta x)^{2}$ under the condition $r=1+O(\Delta x)$. It follows that the truncation error in (14) is reduced to $O(\Delta x)^{4}$, provided that the condition of $r=1+O(\Delta x)$ is satisfied.

From the foregoing, we can establish

$$
\begin{aligned}
\frac{\delta^{2} E_{i}}{\Delta x^{2}}= & R_{1}\left(\sigma \frac{\partial E}{\partial z}-\nu E\right)_{i+1}+R_{2}\left(\sigma \frac{\partial E}{\partial z}-\nu E\right)_{i} \\
& +R_{3}\left(\sigma \frac{\partial E}{\partial z}-\nu E\right)_{i-1} \\
R_{1}= & \frac{3 r^{2}-3 r+1}{6 r(r+1)} \\
R_{2}= & \frac{-r^{2}+7 r-1}{6 r} \\
R_{3}= & \frac{r^{2}-3 r+3}{6(r+1)} .
\end{aligned}
$$

Alternatively, we can replace $\delta$ in (13) by the following three-point representation:

$$
\delta E_{i}=\frac{\left(E_{i+1}-E_{i}\right) / r+r\left(E_{i}-E_{i-1}\right)}{r+1} .
$$

In this case, we obtain the slightly different coefficients

$$
\begin{aligned}
& R_{1}=\frac{r^{2}+r-1}{6 r(r+1)} \\
& R_{2}=\frac{r^{2}+3 r+1}{6 r} \\
& R_{3}=\frac{-r^{2}+r+1}{6(r+1)} .
\end{aligned}
$$

As can be expected, (18) is reduced to the uniform case when $r=1$ in [26, eq. (3)]. After some manipulations, we finally 
construct the finite-difference equations.

$$
\begin{aligned}
& \phi_{i+1}^{-} E_{i+1}^{m+1}+\chi_{i}^{-} E_{i}^{m+1}+\psi_{i-1}^{-} E_{i-1}^{m+1} \\
& \quad=\phi_{i+1}^{+} E_{i+1}^{m}+\chi_{i}^{+} E_{i}^{m}+\psi_{i-1}^{+} E_{i-1}^{m} \\
& \phi_{i+1}^{ \pm}=R_{1} \sigma \pm \frac{\Delta z}{r(r+1) \Delta x^{2}} \pm \frac{\Delta z}{2} R_{1} \nu_{i+1} \\
& \chi_{i}^{ \pm}=R_{2} \sigma \mp \frac{\Delta z}{r \Delta x^{2}} \pm \frac{\Delta z}{2} R_{2} \nu_{i} \\
& \psi_{i-1}^{ \pm}=R_{3} \sigma \pm \frac{\Delta z}{(r+1) \Delta x^{2}} \pm \frac{\Delta z}{2} R_{3} \nu_{i-1} .
\end{aligned}
$$

We further extend the formulation to the multistep method. As in the case of (7), the $N$-step algorithm may be written in the form

$$
\frac{\partial E}{\partial z}=\frac{2 j \operatorname{Im}\left\{a_{K}\right\} P}{\left(1+\operatorname{Re}\left\{a_{K}\right\} P\right) \Delta z / N} E
$$

where

$$
P=\frac{\partial^{2}}{\partial x^{2}}+\nu
$$

Now we apply (18) to (23), so that the following finitedifference equation is derived:

$$
\begin{aligned}
& \zeta_{i+1}^{m+K / N} E_{i+1}^{m+K / N}+\xi_{i}^{m+K / N} E_{i}^{m+K / N}+\eta_{i-1}^{m+K / N} E_{i-1}^{m+K / N} \\
& \quad=\zeta_{i+1}^{m+(K-1) / N} E_{i+1}^{m+(K-1) / N}+\xi_{i}^{m+(K-1) / N} E_{i}^{m+(K-1) / N} \\
& \quad+\eta_{i-1}^{m+(K-1) / N} E_{i-1}^{m+(K-1) / N}
\end{aligned}
$$

where

$$
\begin{aligned}
\zeta_{i+1}^{m+(K-1) / N} & =R_{1}+\Gamma_{K}\left[\frac{2}{r(r+1) \Delta x^{2}}+R_{1} \nu_{i+1}^{m+1 / 2}\right] \\
\xi_{i}^{m+(K-1) / N} & =R_{2}-\Gamma_{K}\left[\frac{2}{r \Delta x^{2}}-R_{2} \nu_{i}^{m+1 / 2}\right] \\
\eta_{i-1}^{m+(K-1) / N} & =R_{3}+\Gamma_{K}\left[\frac{2}{(r+1) \Delta x^{2}}+R_{3} \nu_{i-1}^{m+1 / 2}\right] .
\end{aligned}
$$

It should be restressed that (22) and (24) are the six-point schemes which can be solved efficiently. The PSM has an advantage over the CSM in that an arbitrary region in the transverse coordinate can be densely or coarsely discretized. This advantage will be used for an adaptive grid in Section III.

\section{Numerical Results}

To confirm the accuracy of the present methods, we treat a symmetrical graded-index slab waveguide in which the refractive index is $n^{2}(x)=n_{s}^{2}+2 n_{s} \Delta n / \cosh ^{2}(2 x / w)$, where $n_{s}=2.1455, \Delta n=0.003$ and $w=5 \mu \mathrm{m}$. The wavelength considered here is $\lambda=1.3 \mu \mathrm{m}$.

Preliminary calculations show that when the grid growth factor in the PSM is chosen to be the same as that determined by the CSM, both methods yield almost identical results. Therefore, we show only the results for the CSM. The numerical parameters are chosen to be $\alpha=100 \mu \mathrm{m}$ and $|u| \leq \pi / 4$. This corresponds to a computational window dimension of $200 \mu \mathrm{m}$ in the physical space. The transparent boundary condition is imposed at the computational window edges [40].

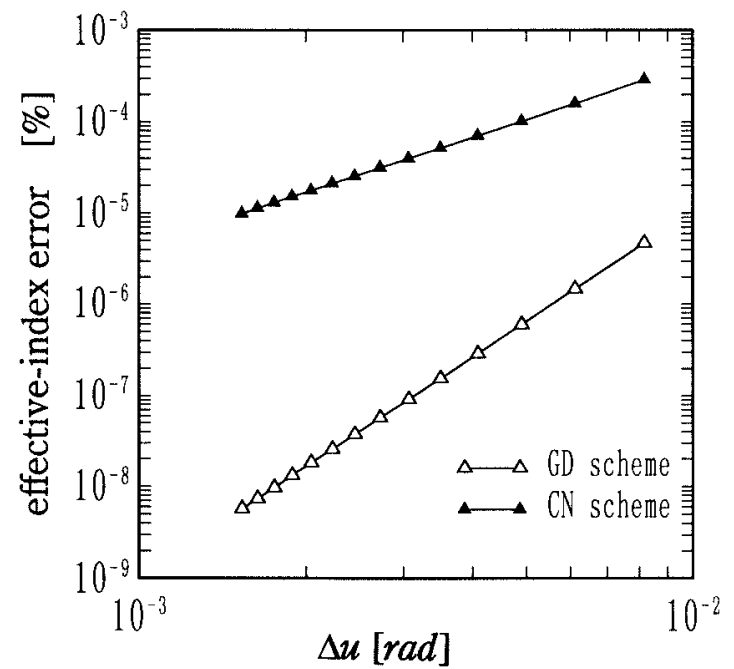

Fig. 2. Effective-index error in a straight waveguide as a function of $\Delta u$.

First, we test the paraxial formulation. The lowest order eigenmode is analyzed using the imaginary distance BPM [37]-[39], in which the effective index $n_{c a l}$ is calculated by the following equation [38]:

$$
n_{c a l}=n_{0}+\frac{\ln \int \mathcal{E}\left(u, z^{\prime}+\Delta z^{\prime}\right) d u-\ln \int \mathcal{E}\left(u, z^{\prime}\right) d u}{k \Delta z^{\prime}}
$$

where $z^{\prime}=-j z$. The effective-index error is defined as

$$
\varepsilon=\frac{n_{c a l}-n_{e x}}{n_{e x}} \times 100[\%]
$$

where $n_{e x}$ is the exact effective index.

Fig. 2 shows the effective-index error of the fundamental mode as a function of the transverse sampling width $\Delta u$. For comparison, the results of the conventional $\mathrm{CN}$ scheme are also shown. A propagation step length of $\Delta z^{\prime}=1.0 \mu \mathrm{m}$ is used. It is noteworthy that the effective index error of the present scheme is almost proportional to $\Delta u^{4}$. In contrast, the error of the conventional $\mathrm{CN}$ scheme is proportional to $\Delta u^{2}$.

Second, we assess the wide-angle formulation. The propagation of the lowest order mode in a tilted waveguide is analyzed. The mode profile of the propagating field is evaluated by the coupling efficiency defined as

$$
\eta=\frac{\left|\int E_{0} E^{*} d x\right|^{2}}{\left(\int\left|E_{0}\right|^{2} d x\right)^{2}}
$$

where $E$ is the propagating field and $E_{0}$ is the incident field of the fundamental mode. Note that the transverse coordinate $u$ is restored to $x$. In the case of no calculation error, the guidedmode field continues to propagate without any distortion, so that the coupling efficiency becomes unity.

Fig. 3 presents the coupling efficiency as a function of tilt angle, in which $\Delta u \simeq 7.854 \times 10^{-4} \mathrm{rad}$ and $\Delta z=0.05 \mu \mathrm{m}$ are used. The results obtained at a propagation distance of $100 \mu \mathrm{m}$ are shown for the paraxial, one-, two-, and three-step cases. It is clearly seen that the GD scheme improves the coupling 


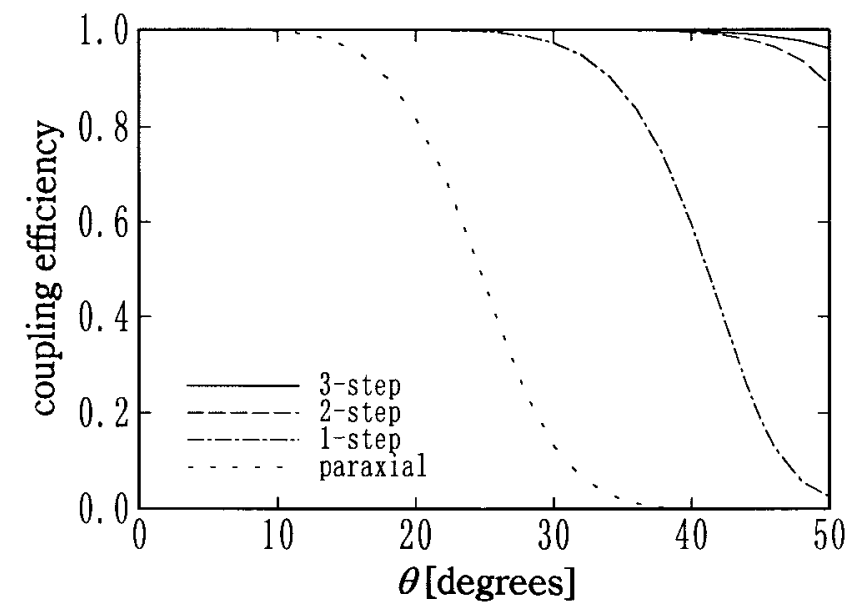

(a)

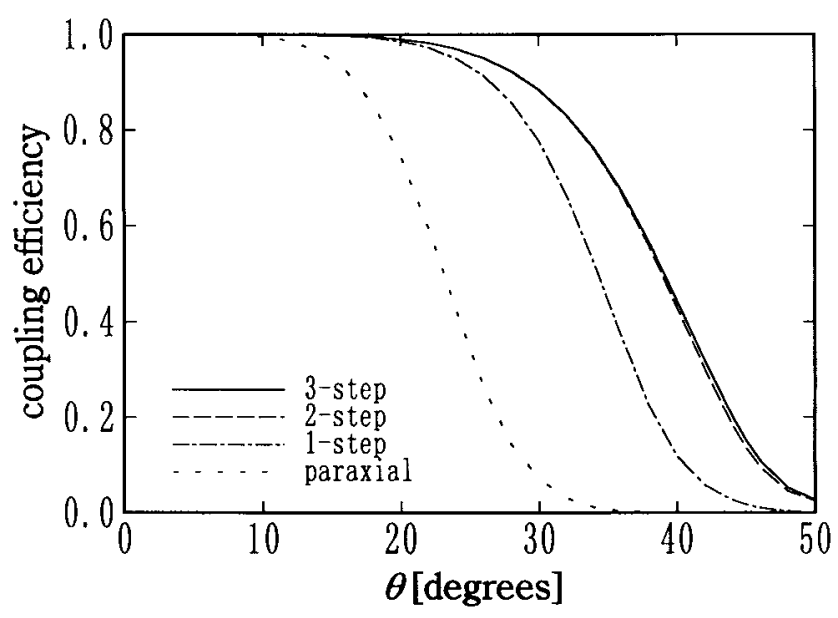

(b)

Fig. 3. Coupling efficiency as a function of tilt angle $\theta$ : (a) GD scheme and (b) $\mathrm{CN}$ scheme.

efficiency, when compared with the conventional CN scheme. To clarify the reason for the improvement of the GD scheme, we illustrate the propagating field obtained for the three-step method in Fig. 4, in which the tilt angle is chosen to be $45^{\circ}$. For comparison, the result obtained from the $\mathrm{CN}$ scheme is also shown. It is seen that the field obtained from the GD scheme propagates with the mode profile being maintained, while that from the $\mathrm{CN}$ scheme not only deforms but also shifts toward the $-x$ direction. Fig. 5 shows the field profile at a propagation distance of $100 \mu \mathrm{m}$. The field distribution for the GD scheme agrees fairly with the exact field, while that for the $\mathrm{CN}$ scheme fails.

\section{ApplicAtion to AN ADAPTive GRID}

Although the CSM is easy to implement as discussed in Section II-A, the grid growth factor in the physical space is limited due to the mapping function to be used. In contrast, the PSM has flexibility in the choice of the grid growth factor. Therefore, we introduce an adaptive grid [18], [19], [21], [22], [24], [25] into the multistep method based on the PSM. Since the numerical results using the coefficients $R$ 's in (19) and (21) do not show significant difference [29], we adopt $R$ 's in (19).

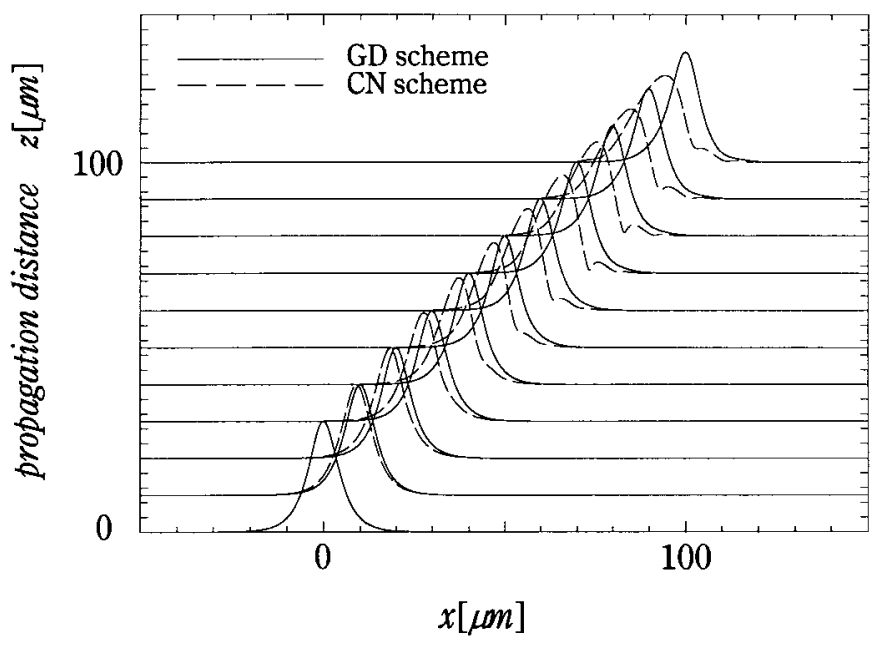

Fig. 4. Propagating field in a tilted slab waveguide. The tilt angle is $45^{\circ}$.

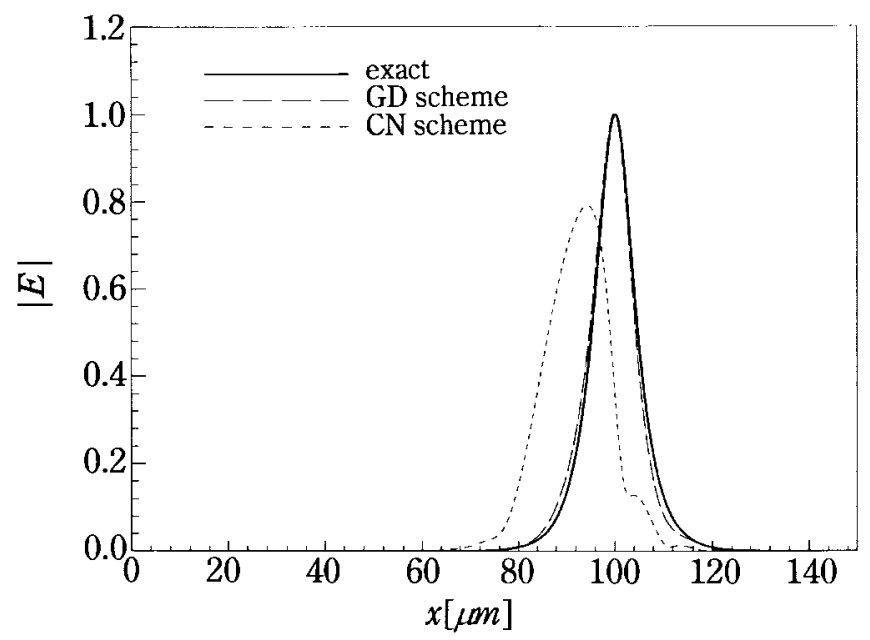

Fig. 5. Field profiles at a propagation distance of $100 \mu \mathrm{m}$. The tilt angle is $45^{\circ}$.

The implementation of an adaptive grid can be generalized using the local amplitude of the field [22]. In this analysis, we add the sampling points in such a way that the initial transverse sampling width is divided into $\Delta x / 2$ in the region where the local amplitude of the field is larger than a fixed value. The missing values between the sampling points are generated using a Lagrange interpolation technique. Fig. 6 shows an example of the distribution of sampling points using the adaptive grid, in which the position of the sampling points varies stepwise along the waveguide.

The waveguide tested here is the same as that discussed in Section II. The tilt angle is varied from 0 to $50^{\circ}$. The coupling efficiency of the fundamental mode evaluated at a propagation distance of $100 \mu \mathrm{m}$ is shown in Fig. 7, in which the threestep method is used and the total number of sampling points is designated as $N_{x}$. The sampling widths are $\Delta x=0.2 \mu \mathrm{m}$ and $\Delta z=0.05 \mu \mathrm{m}$. It is found that the multistep method with an adaptive grid can successfully achieve high accuracy regardless of a small number of sampling points. In the regular grid, 2000 sampling points are needed to obtain the same accuracy as that for the adaptive grid. 


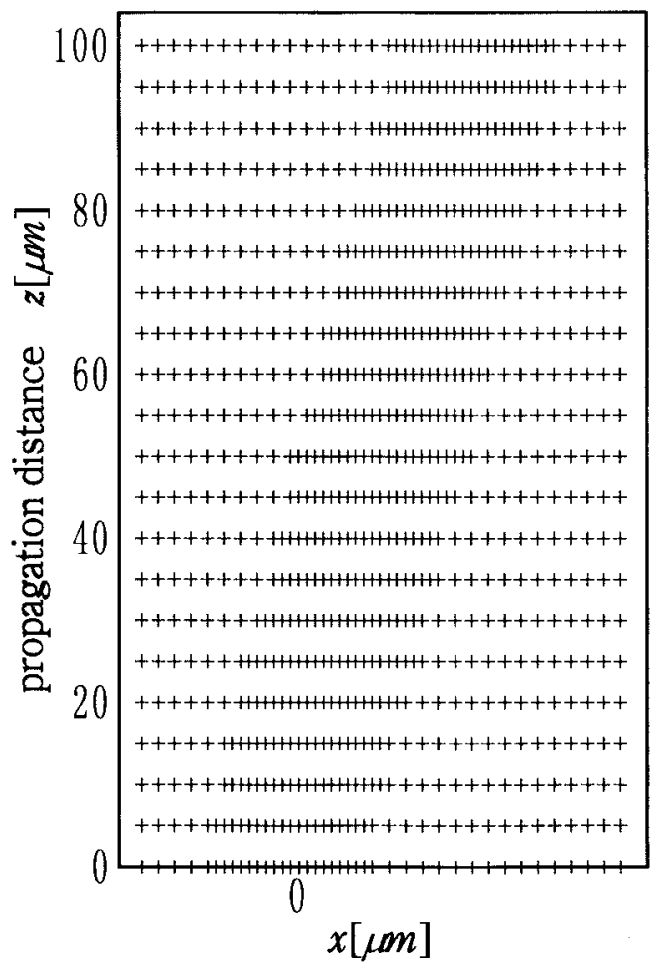

Fig. 6. Example of an adaptive grid for a tilted waveguide.

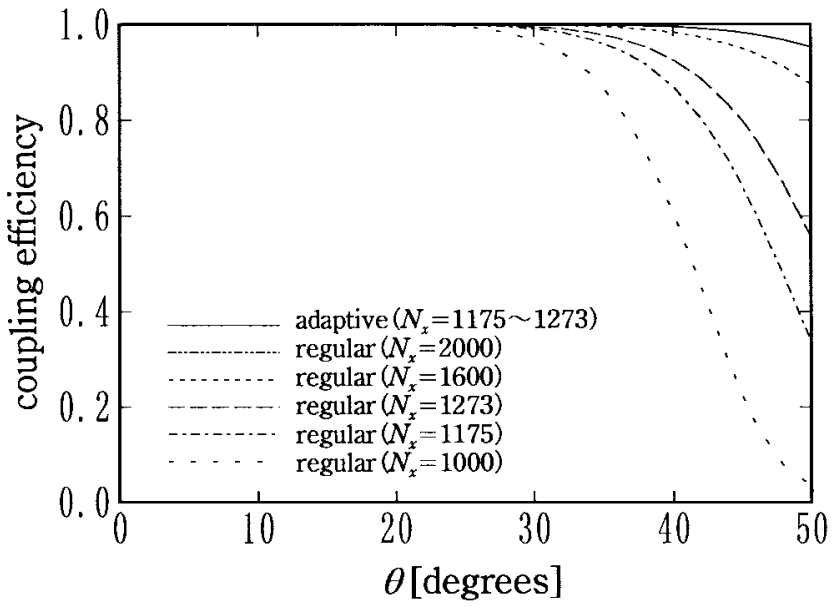

Fig. 7. Comparison between adaptive and regular grids in coupling efficiency as a function of tilt angle $\theta$.

\section{CONCLUSIONS}

We have studied the applicability of the high-accuracy nonuniform schemes based on the generalized Douglas scheme to the FD-BPM. The fourth-order accuracy is achieved in the transverse direction, provided that the grid growth factor between two adjacent grids is $r=1+O(\Delta x)$. In a twodimensional problem, two different methods are derived. For the computational space method, a mapping technique is used to transform a nonuniform grid in the physical space into a uniform one in the computational space. The mapping function used in this paper is particularly suitable for a modal analysis of an optical waveguide. This method is easy to implement with only slight modification to the conventional algorithm. For the physical space method, the GD scheme is directly extended to a nonuniform grid by using a Taylorseries expansion. The latter method is superior to the former one in that we have flexibility in the choice of the grid growth factor: an arbitrary region in the transverse coordinate can be densely or coarsely discretized. Several numerical results show the effectiveness of the present methods for both the paraxial and wide-angle formulations. Finally, taking advantage of the physical space method, we introduce an adaptive grid into the multistep method. With the aid of the adaptive grid, the total number of the transverse sampling points can be substantially reduced, without losing inherent accuracy.

\section{ACKNOWLEDGMENT}

The authors would like to thank Dr. G. R. Hadley of Sandia National Laboratories for invaluable discussions on a highaccuracy scheme.

\section{REFERENCES}

[1] D. Yevick, "A guide to electric field propagation techniques for guidedwave optics," Opt. Quantum Electron., vol. 26, pp. S185-S197, 1994.

[2] H. J. W. M. Hoekstra, "On beam propagation methods for modeling in integrated optics," Opt. Quantum Electron., vol. 29, pp. 157-171, 1997.

[3] M. D. Feit and J. A. Fleck, Jr., "Light propagation in graded-index optical fibers," Appl. Opt., vol. 17, pp. 3990-3998, 1978.

[4] D. Yevick and B. Hermansson, "Split-step finite difference analysis of rib waveguides," Electron. Lett., vol. 25, pp. 461-462, 1989.

[5] Y. Chung and N. Dagli, "An assessment of finite difference beam propagation method," IEEE J. Quantum Electron., vol. 26, pp. 1335-1339, 1990.

[6] R. Accornero, M. Artiglia, G. Coppa, P. Di Vita, G. Lapenta, M. Potenza, and P. Ravetto, "Finite difference methods for the analysis of integrated optical waveguides," Electron. Lett., vol. 26, pp. 1959-1960, 1990.

[7] R. Scarmozzino and R. M. Osgood, Jr., "Comparison of finite-difference and Fourier-transform solutions for the parabolic wave equation with emphasis on integrated-optics applications," J. Opt. Soc. Amer. A, vol. 8, pp. 724-731, 1991.

[8] D. Yevick and M. Glasner, "Forward wide-angle light propagation in semiconductor rib waveguides," Opt. Lett., vol. 15, pp. 174-176, 1990.

[9] G. R. Hadley, "Wide-angle beam propagation using Padé approximant operators," Opt. Lett., vol. 17, pp. 1426-1428, 1992.

[10] F. Ma, C. L. Xu, and W. P. Huang, "Wide-angle full vectorial beam propagation method," Inst. Elec. Eng. Proc.-Optoelectron., vol. 143, pp. 139-143, 1996.

[11] T. B. Koch, J. B. Davies, and D. Wickramasinghe, "Finite element/finite difference propagation algorithm for integrated optical device," Electron. Lett., vol. 25, pp. 514-516, 1989.

[12] K. Hayata, A. Misawa, and M. Koshiba, "Split-step finite-element method applied to nonlinear integrated optics," J. Opt. Soc. Amer. B, vol. 7, pp. 1772-1784, 1990.

[13] Y. Arai, A. Maruta, and M. Matsuhara, "Transparent boundary for the finite-element beam-propagation method," Opt. Lett., vol. 18, pp. 765-766, 1993.

[14] H. E. Hernández-Figueroa, "Simple nonparaxial beam-propagation method for integrated optics," J. Lightwave Technol., vol. 12, pp. 644-649, 1994

[15] J. Gerdes and R. Pregla, "Beam-propagation algorithm based on the method of lines," J. Opt. Soc. Amer. B, vol. 8, pp. 389-394, 1991.

[16] D. Yevick, J. Yu, M. Munowitz, and D. Vezzetti, "Modal analyses of semiconductor rib waveguides employing nonequidistant grids," J. Opt. Soc. Amer., vol. 8, pp. 1385-1388, 1991.

[17] Y. Chung and N. Dagli, "Analysis of Z-invariant and Z-variant semiconductor rib waveguides by explicit finite difference beam propagation method with nonuniform mesh configuration," IEEE J. Quantum Electron., vol. 27, pp. 2296-2305, 1991.

[18] M. Artiglia, P. Di Vita, M. Potenza, G. Coppa, G. Lapenta, and P. Ravetto, "Variable grid finite difference methods for study of longitudinally varying planar waveguides," Electron. Lett., vol. 27, pp. 474-475, 1991.

[19] T. Rasmussen, J. H. Povlsen, and A. Bjarklev, "Accurate finite difference beam propagation method for complex integrated optical structures," IEEE Photon. Technol. Lett., vol. 5, pp. 339-342, 1993. 
[20] P. L. Liu, S. L. Yang, and D. M. Yuan, "The semivectorial beam propagation method," IEEE J. Quantum Electron., vol. 29, pp. 1205-1211, 1993.

[21] C. Massini, G. Bellanca, P. Bassi, and R. Sorrentino, "An FD-BPM with adaptive mesh for longitudinally varying optical structures," Opt. Quantum Electron., vol. 27, pp. 951-959, 1995.

[22] F. Schmidt, "An adaptive approach to the numerical solution of Fresnel's wave equation," J. Lightwave Technol., vol. 11, pp. 1425-1434, 1993.

[23] G. S. Pu, T. Mizumoto, and Y. Naito, "Modified numerical technique for beam propagation method based on the Galerkin's technique," IEICE Trans. Electron., vol. E77-C, pp. 510-514, 1994.

[24] M. Koshiba and Y. Tsuji, "A wide-angle finite-element beam propagation method," IEEE Photon. Technol. Lett., vol. 8, pp. 1208-1210, 1996.

[25] Y. Tsuji, M. Koshiba, and T. Tanabe, "A wide-angle beam propagation method using a finite element scheme," IEICE Trans., vol. J79-C-I, pp. 381-388, 1996.

[26] J. Yamauchi, J. Shibayama, and H. Nakano, "Modified finite-difference beam propagation method based on the generalized Douglas scheme for variable coefficients," IEEE Photon. Technol. Lett., vol. 7, pp. 661-663, 1995.

[27] J. Yamauchi, J. Shibayama, O. Saito, O. Uchiyama, and H. Nakano, "Improved finite-difference beam-propagation method based on the generalized Douglas scheme and its application to semivectorial analysis," J. Lightwave Technol., vol. 14, pp. 2401-2406, 1996.

[28] J. Yamauchi, J. Shibayama, and H. Nakano, "Wide-angle propagating beam analysis based on the generalized Douglas scheme for variable coefficients," Opt. Lett., vol. 20, pp. 7-9, 1995.

[29] J. Yamauchi, J. Shibayama, M. Sekiguchi, and H. Nakano, "Finitedifference beam propagation method based on the generalized Douglas scheme for a nonuniform grid," IEEE Photon. Technol. Lett., vol. 9, pp. 67-69, 1997.

[30] C. Vassallo and M. J. Van der Keur, "Highly efficient transparent boundary conditions for finite difference beam propagation method at order four," J. Lightwave Technol., vol. 15, pp. 1958-1965, 1997.

[31] F. Ladouceur, "Boundaryless beam propagation," Opt. Lett., vol. 21, pp. 4-5, 1996.

[32] S. J. Hewlett and F. Ladouceur, "Fourier decomposition method applied to mapped infinite domains: Scalar analysis of dielectric waveguides down to modal cutoff," J. Lightwave Technol., vol. 13, pp. 375-383, 1995.

[33] K. M. Lo and E. H. Li, "Solutions of the quasivector wave equation for optical waveguides in a mapped infinite domains by the Galerkin's method," J. Lightwave Technol., vol. 16, pp. 937-944, 1998.

[34] G. R. Hadley, "Multistep method for wide-angle beam propagation," Opt. Lett., vol. 17, pp. 1743-1745, 1992.

[35] J. Yamauchi, J. Shibayama, M. Sekiguchi, and H. Nakano, "Improved multistep method for wide-angle beam propagation," IEEE Photon. Technol. Lett., vol. 8, pp. 1361-1363, 1996.

[36] O. Mitomi and K. Kasaya, "Wide-angle finite-element beam propagation method using Padé approximation," Electron. Lett., vol. 33, pp. 1461-1462, 1997

[37] D. Yevick and W. Bardyszewski, "Correspondence of variational finitedifference (relaxation) and imaginary-distance propagation methods for modal analysis," Opt. Lett., vol. 17, pp. 329-330, 1992.

[38] C. L. Xu, W. P. Huang, and S. K. Chaudhuri, "Efficient and accurate vector mode calculations by beam propagation method," J. Lightwave Technol., vol. 11, pp. 1209-1215, 1993.

[39] S. Jüngling and J. C. Chen, "A study and optimization of eigenmode calculations using imaginary-distance beam-propagation method," IEEE J. Quantum Electron., vol. 30, pp. 2098-2105, 1994.

[40] G. R. Hadley, "Transparent boundary condition for beam propagation," Opt. Lett., vol. 16, pp. 624-626, 1991.

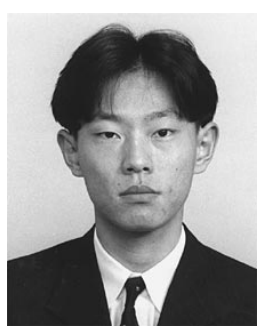

(IEICE) of Japan

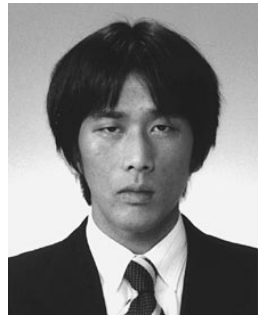

Kenji Matsubara was born in Tokyo, Japan, on September 4, 1974. He received the B.E. degree from Hosei University, Tokyo, Japan, in 1997 and is currently pursuing the M.E. degree.

Mr. Matsubara is a member of the Institute of Electronics, Information and Communication Engineers (IEICE) of Japan.

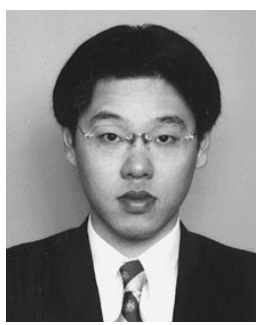

Minoru Sekiguchi was born in Ibaraki, Japan, on February 10, 1973. He received the B.E. and M.E degrees from Hosei University, Tokyo, Japan, in 1995 and 1997, respectively.

In 1997, he joined Hitachi Co., Ltd., and is currently with the College of Engineering, Hosei University, Tokyo, Japan.

Mr. Sekiguchi is a member of the Institute of Electronics, Information and Communication Engineers of Japan.

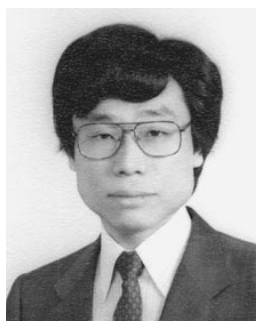

Junji Yamauchi (M'85) was born in Nagoya, Japan, on August 23, 1953. He received the B.E., M.E., and Dr.E. degrees from Hosei University, Tokyo, Japan, in 1976, 1978, and 1982, respectively.

From 1984 to 1988 , he served as a Lecturer in the Electrical Engineering Department of Tokyo Metropolitan Technical College. Since 1988, he has been a member of the faculty of Hosei University, where he is now a Professor of electronic informatics. His research interests include optical waveguides and circularly polarized antennas.

Dr. Yamauchi is a member of the Optical Society of America (OSA) and the Institute of Electronics, Information and Communication Engineers (IEICE) of Japan.

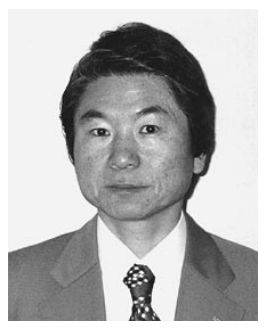

Hisamatsu Nakano (M'75-SM'87-F'92) was born in Ibaraki, Japan, on April 13, 1945. He received the B.E., M.E., and Dr.E. degrees in electrical engineering from Hosei University, Tokyo, Japan, in 1968,1970 , and 1974 , respectively.

Since 1973, he has been a member of the faculty of Hosei University, where he is now a Professor of Electronic Informatics. His research topics include numerical methods for antennas, electromagnetic wave scattering problems, and lightwave problems. He has published more than 130 refereed journal papers and 90 international symposium papers on antenna and relevant problems. He is the author of Helical and Spiral Antennas (New York: Research Studies Press, Wiley, 1987). He published the chapter "Antenna Analysis using Integral Equations," in Analysis Methods of Electromagnetic Wave Problems, vol. 2 (Norwood, MA: Artech House, 1996). He was a Visiting Associate Professor at Syracuse University, Syracuse, NY, during May-September 1981, a Visiting Professor at University of Manitoba, Canada, during March-September 1986, and a Visiting Professor at the University of California, Los Angeles, during September 1986-March 1987.

Dr. Nakano received an International Scientific Exchange Award from the Natural Sciences and Engineering Research Council of Canada. In 1987, he received the Best Paper Award from the IEE 5th International Conference on Antennas and Propagation. In 1994, he received the IEEE Antennas and Propagation Society (AP-S) Best Application Paper Award (H. A. Wheeler Award). $\mathrm{He}$ is an Associate Editor of IEEE Antennas AND Propagation MAGAZINE. 